\title{
Considerations on finding the rolling and spinning friction coefficients
}

\author{
Florina-Carmen Ciornei ${ }^{1, *}$ Stelian Alaci ${ }^{1}$, Sorinel-Toderas Siretean ${ }^{1}$, Mariana-Catalina Ciornei $^{2}$, \\ Ioan-Bogdan Dragoi ${ }^{1}$ and Vlădut-Constantin Lazăr ${ }^{1}$ \\ ${ }^{1}$ Stefan cel Mare University, Suceava, 13 University Str., 720229, Romania \\ ${ }^{2}$ Carol Davila University of Medicine and Pharmacy, Dpt. Clinic 2, 8 Bd. Eroii Sanitari, 050474, Bucharest, Romania
}

\begin{abstract}
The complex motion from a point contact has as consequence the occurrence of a friction torsor within both spinning and rolling friction exist. Additionally, recent researches showed that the two friction moments, spinning and rolling torques, are not proportional to the normal force. Here, the power law dependency is accepted and therefore, besides the coefficients of spinning and rolling friction, the exponents from the relations friction torque-normal force should be first determined. The paper proposes as method for finding the four parameters the use of the inclined plane principle. The acceleration of a revolution body in descending motion on the inclined plane is found for four different values of the tilting angle and a system of four equations is obtained. The detailed procedure of finding the solutions of the system is presented in the present paper.
\end{abstract}

\section{Introduction}

The Hertzian type contacts are frequently met in machine building in diverse systems such as: cam mechanisms, gear transmissions, elasto-hydrodynamic variators etc. These mechanical contacts are characterized by the existence between the two bodies of a sliding motion described by the sliding velocity $\boldsymbol{v}_{a l}$, contained in the common tangent plane $(P)$ and a rotation motion, the last described by the relative angular velocity $\omega$ with a component about the common normal - the spinning angular velocity $\omega_{s}$, and another component contained in the tangent plane - the rolling angular velocity $\omega_{r}$. The presence of the relative motion in the nonconforming contact generates a friction torsor, composed by: the friction force $T$ collinear with the relative velocity $\boldsymbol{v}_{a l}$ but of opposite sense; the spinning friction torque $\boldsymbol{M}_{S}$, collinear and of opposite sense to the angular spinning velocity and the rolling friction torque, $\boldsymbol{M}_{r},[1]$.

The precise evaluation of the components of the friction torsor is a fundamental task in tribological research since the values of these parameters together to the relative motions are directly in control of the energy losses from a mechanical transmission, losses revealed by the value of the mechanical efficiency of the transmission, [2].

Concerning the velocity and the sliding friction $\boldsymbol{T}$, the theory is well established both for lubricated contacts by the theory of elasto-hydrodynamic lubrication and for dry friction contacts, by the theory based on the AmontonCoulomb laws, [3]; as for spinning and rolling friction torques, the theory is an open topic and is the subject of numerous scientific works and monographs.

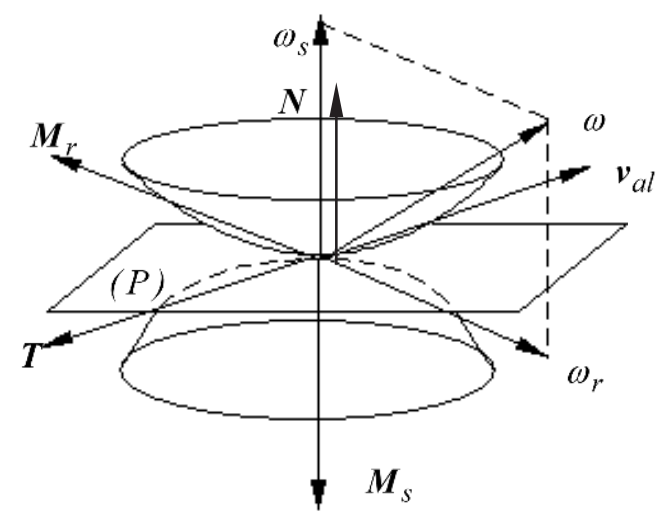

Fig. 1. The relative motions and the elements of the friction torsor in a Hertzian contact

The dynamical treatises accept, in a first approximation, analogous to the case of sliding friction, that both the spinning and rolling friction torque are proportional to the normal force from the contact $N$, the proportionality factors being the coefficient of spinning friction $s_{S}$ and the coefficient of rolling friction $s_{r}$ respectively:

$$
\begin{aligned}
& M_{S}=s_{S} N \\
& M_{r}=s_{r} N
\end{aligned}
$$

\footnotetext{
* Corresponding author: florina@fim.usv.ro
} 
Recent papers based on elements from the theory of elasticity conclude that equally the spinning friction torque Popov [4] and the rolling friction torque Chjerepanov [5] present a dependency on the normal force of power law type, specifically:

$$
M_{s}=s_{S} N^{\alpha_{s}}
$$

and

$$
M_{r}=s_{r} N^{\alpha_{r}}
$$

Under this power-law dependency the tribological and energetically study is obviously more complicated given that not only the coefficients $s_{S}$ and $s_{r}$ must be found, but also the exponents $\alpha_{s}$ and $\alpha_{r}$ from the relations 3 and 4. In a series of recent works, [6-10], there were presented several methods and the devices used in the estimation of either the coefficient of spinning friction or of the coefficient of rolling friction.

As principle, these techniques employ oscillatory motions between the contacting surfaces or continuous motions. From the schemes based on monotonous velocity motions, one of the most employed is the inclined plane method. But one of the major drawbacks of the method resides in the extremely small value of the slope of the plane. This method gives precise results when the ratio $s_{r} / r$ (where $r$ is the rolling radius) is comparable to the inclination angle $\gamma$ of the plane.

$$
s_{r} / r \cong \gamma[\mathrm{rad}]
$$

When the condition 5 is disobeyed, the results are comparable to the measuring errors. Assuming non-linear dependence between the friction torques and the normal force, the relations 3 and 4 , the principle of superposition from linear elasticity cannot be applied and therefore, there are required experimental techniques which allow for simultaneous finding of the characteristic friction parameters of spinning and rolling.

\section{The principle of the proposed method}

The need of synchronized estimation of spinning and rolling friction torques conducted to the method and device [11] consisting in an inclined conduct (groove) with respect to horizontal plane. A bearing ball set on the top of the V-groove, contacting both walls, is let into to free motion and the time necessary for running an imposed length along the channel permits finding the acceleration and implicitly gives information about the spinning friction and rolling friction between the wall and the ball.

Accepting linear dependency between the friction torques and the normal force, two experiments carried out for two slopes of the groove are required. The method was provisionally neglected, due to the impossibility of precise controlling of the tilting angle.

The present paper analyses the possibility of finding the characteristic parameters of spinning friction and rolling friction under the assumption of nonlinear dependency on the normal force.

The principle of the method is presented in Fig. 2. The operating principle of the test rig is relatively simple. A bearing ball of $R$ radius that has a cylindrical ring attached to the equatorial zone is set on top of two cylindrical parallel rods. The parallelism between the axes of the rods is accurately controlled with two prismatic parts having each two holes of diameters equal to the ones of the rods. The holes from the two parts are machined simultaneously in order to have precisely the same distance between axes. The horizontality of the plane containing the rods can be controlled with accuracy by placing a ball in contact with the two rods. When the balls are immobile, the plane of their axes can be considered horizontal. A cord winded over the ring passes over a pulley of negligible mass.

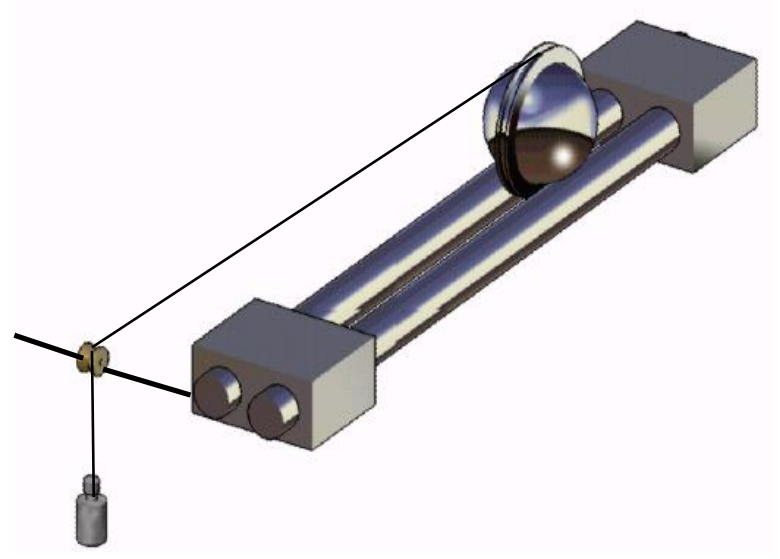

Fig. 2. The principle of the experimental device

For the dynamical analysis, the scheme from Fig. 3 is considered. A body with the mass $m$ is hanging at the end of the cord and has the function of actuating the ball into motion. The ball is contacting the rods in two points, $C^{\prime}$ and $C^{\prime \prime}$. Assuming that there is no slip in these points, the straight line $C^{\prime} C^{\prime \prime}$ is the instantaneous axis of rotation and the angular velocity is parallel to it.

In each of the two contact points occur: the friction force, $\boldsymbol{T}^{\prime}, \boldsymbol{T}^{\prime \prime}$ parallel to the $O x$ axis; the moment of spinning friction $\boldsymbol{M}_{S}^{\prime}, \boldsymbol{M}_{s}^{\prime \prime}$ directed along the normal on the contact point and the moment of rolling friction $\boldsymbol{M}_{r}^{\prime}, \boldsymbol{M}_{r}^{\prime \prime}$ normal to the $\boldsymbol{M}_{s}^{\prime}, \boldsymbol{M}^{\prime \prime}{ }_{s}$, respectively. Applying the hypothesis of dry friction, all the components of the friction torsor from the two contact points are constant and thus it is expected that the motion of the ball is uniformly accelerated.

The theorem of motion of center of mass and the moment of momentum theorem are applied with respect to the center of mass in order to find the law of motion of the ball.

The center of mass moves along the horizontal direction and the abscissa $x_{G}$ describes its position. The ball also has a rotation motion characterized by the angle $\varphi$. It is considered that initially: 


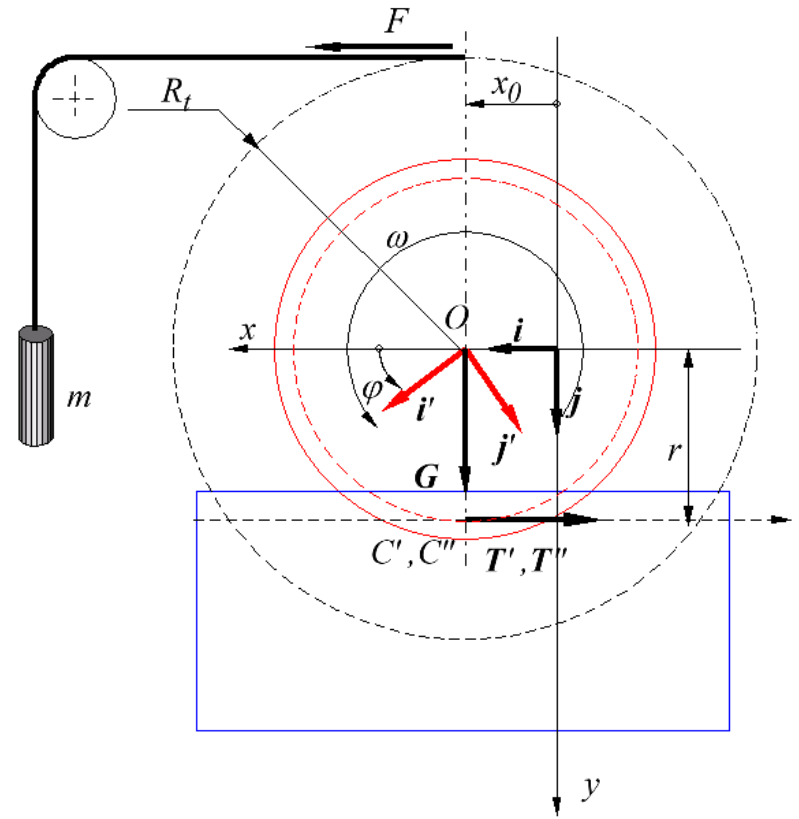

Fig. 3. The schematics for dynamical analysis

$$
x=0, \varphi=0
$$

The pure rolling condition is expressed as:

$$
x_{G}=r \varphi
$$

where $r$ is the distance from the center of the ball to the line of the contact points $C^{\prime} C^{\prime \prime}$. Thus, one of the parameters $\varphi$ or $x_{G}$ is sufficient to characterize the position of the ball. The equation of motion of the center of the ball has the following form:

$$
M \ddot{r}_{G}=\boldsymbol{G}+\boldsymbol{N}^{\prime}+\boldsymbol{N}^{\prime \prime}+\boldsymbol{T}^{\prime}+\boldsymbol{T}^{\prime \prime}+\boldsymbol{F}
$$

where $\boldsymbol{F}$ is the tension from the rope, equal in modulus with:

$$
F=m g
$$

Due to symmetry, the following equalities are valid:

$$
N^{\prime}=N^{\prime \prime}, T^{\prime}=T^{\prime \prime}
$$

and are considered in the projections of the equation 8 on the axes. It results the following system:

$$
\begin{aligned}
M r \varepsilon & =m g-2 T \\
0 & =2 N \cos \beta-M g
\end{aligned}
$$

And the following expressions result:

$$
\begin{aligned}
& T=\frac{1}{2} m g-\frac{1}{2} M r \varepsilon \\
& N=\frac{1}{2} M g \cos \beta
\end{aligned}
$$

Based on Fig. 1, the angle can be expressed as

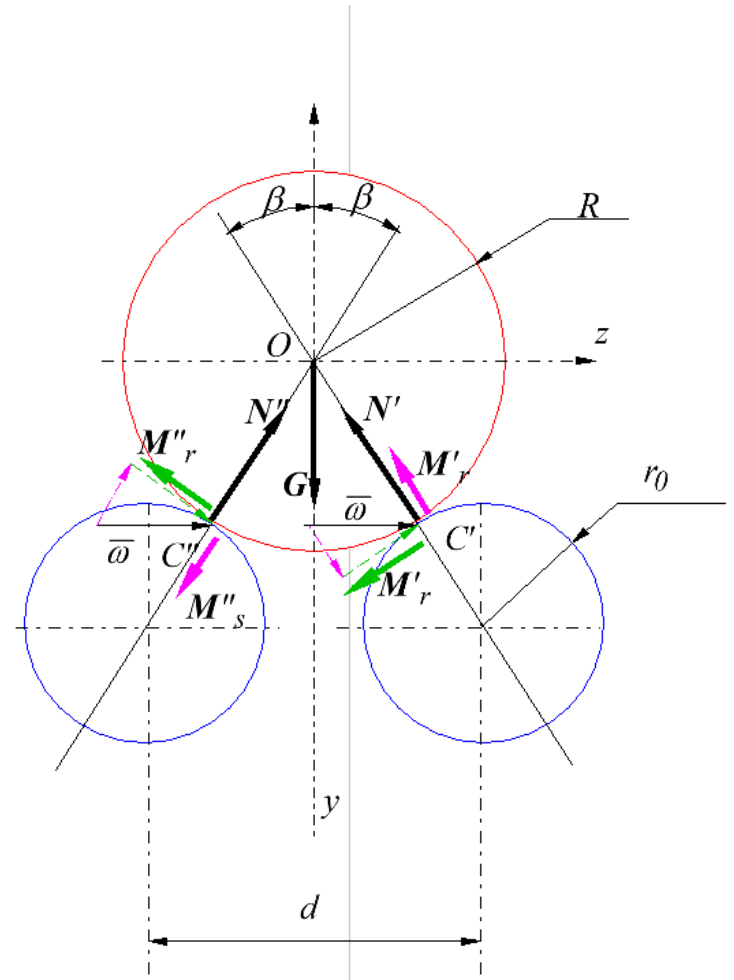

$$
\beta=\operatorname{asin} \frac{d}{2\left(R+r_{0}\right)}
$$

where $r_{0}$ is the radius of the rods. The moment of momentum theorem, expressed as:

$$
\begin{aligned}
& J \boldsymbol{k} \boldsymbol{k}=\boldsymbol{r}_{C^{\prime}} \times\left(\boldsymbol{N}^{\prime}+\boldsymbol{T}^{\prime}\right)+\boldsymbol{r}_{C^{\prime \prime}} \times\left(\boldsymbol{N}^{\prime}+\boldsymbol{T}^{\prime \prime}\right) \\
& +m g R_{t} \boldsymbol{k}+\boldsymbol{M}^{\prime}{ }_{S}+\boldsymbol{M}^{\prime \prime}{ }_{S}+\boldsymbol{M}_{r}^{\prime}+\boldsymbol{M}_{r}^{\prime \prime}{ }_{r}
\end{aligned}
$$

where $R_{t}$ is the radius of winding of the ring, has projection only on $\mathrm{Oz}$ axis. Based on the scheme from Fig. 2, the following scalar equation is obtained:

$$
\varepsilon=\frac{m g R_{t}+2 r T \cos \beta-2 M_{t} \sin \beta-2 M_{r} \sin \beta}{J_{G}}
$$

In the equation 15 there are introduced the proposed dependencies 3 and 4 for the two friction torques and the relation 12 for the modulus of the friction force; the final form of the equation of motion of the ball is obtained:

$$
\varepsilon=\frac{\left[\begin{array}{l}
m g R \cos ^{2} \beta-2 s_{s}\left(\frac{M g}{2 \cos \beta}\right)^{\alpha_{s}} \sin \beta \\
-2 s_{r}\left(\frac{M g}{2 \cos \beta}\right)^{\alpha_{r}} \cos \beta+m g R_{t}
\end{array}\right]}{J_{G}+M R^{2} \cos \beta^{3}}
$$

The relation 16 can now be regarded as a constraining relationship linking the parameters $s_{r}, s_{s}, \alpha_{r}, \alpha_{s}$ : 


$$
\begin{aligned}
& \varepsilon\left(J_{G}+M R^{2} \cos \beta^{3}\right)+2 s_{S}\left(\frac{M g}{2 \cos \beta}\right)^{\alpha_{s}} \sin \beta \\
& +2 s_{r}\left(\frac{M g}{2 \cos \beta}\right)^{\alpha_{r}} \cos \beta-m g R \cos ^{2} \beta=0
\end{aligned}
$$

\section{Discussions concerning the possibilities of finding the tribological parameters}

As affirmed previously, the experimental tests aim to find the parameters $s_{s}, \ldots, \alpha_{s}$ and $\alpha_{r}$ and these will permit establishing the spinning friction torque and rolling friction torque, $M_{s}$ and $M_{r}$, respectively. It results that four tests with different values of the parameters involved in equation 17 are necessary. As it can be noticed, the constructive parameters of the device that can be varied are the angle $\beta$ and the mass $m$ of the actuating weight. The design of the device allows for accurate adjustment by using prismatic parts for supporting the rods with different distances between the guide-holes. Four equations having the following form are obtained:

$$
\begin{gathered}
\varepsilon_{k}\left(J_{G}+M R^{2} \cos \beta_{k}{ }^{3}\right)+2 s_{s}\left(\frac{M g}{2 \cos \beta}\right)^{\alpha_{s}} \sin \beta_{k} \\
\left.+2 s_{r}\left(\frac{M g}{2 \cos \beta_{k}}\right)^{\alpha_{r}} \cos \beta_{k}-m_{k} g R \cos ^{2} \beta_{k}=0, \quad\right) \\
k=1 \div 4
\end{gathered}
$$

The system 18 is system of transcendental equations and for solving it the use of a numerical method (NewtonRaphson) is required. The calculus can be simplified if it considered the notice that the equations 18 are linear in $s_{s}$ and $s_{r}$. Therefore, from the last two relations, $s_{s}$ and $s_{r}$ are expressed as functions of $\alpha_{s}$ and $\alpha_{r}$.

$$
\begin{aligned}
& s_{s}=s_{s}\left(\alpha_{r}, \alpha_{s}\right) \\
& s_{r}=s_{r}\left(\alpha_{s}, \alpha_{r}\right)
\end{aligned}
$$

Due to lack of space the explicit form of the equation 19 is not presented. The equations 19 are introduced in the first two equations 18 and it results a system of transcendental equations in $\alpha_{s}, \alpha_{r}$ having the form:

$$
\begin{aligned}
& H_{k}\left(\alpha_{s}, \alpha_{r}\right)=\varepsilon_{k}\left(J_{G}+M R^{2} \cos \beta_{k}{ }^{3}\right)-m_{k} g R \cos ^{2} \beta_{k} \\
& 2 s_{s}\left(\alpha_{s}, \alpha_{r}\right)\left(\frac{M g}{2 \cos \beta}\right)^{\alpha_{s}} \sin \beta_{k} \\
& +2 s_{r}\left(\alpha_{s}, \alpha_{r}\right)\left(\frac{M g}{2 \cos \beta_{k}}\right)^{\alpha_{r}} \cos \beta_{k}=0 k=1,2
\end{aligned}
$$

The numerical solution of the system 20 written in the simplified manner:

$$
\left\{\begin{array}{l}
H_{1}\left(\alpha_{s}, \alpha_{r}\right)=0 \\
H_{2}\left(\alpha_{s}, \alpha_{r}\right)=0
\end{array}\right.
$$

was strongly dependant on the precision of experimental finding of the angular acceleration $\varepsilon_{k}$.

To illustrate this affirmation, the following example is given. For the parameters of the rotor $R=0.03 \mathrm{~m}$, $R_{t}=0.033 \mathrm{~m}, M=0.882 \mathrm{~kg}$ and $J_{G}=3.493 \mathrm{~kg} \cdot \mathrm{m}^{2}$, the desired parameters were assumed known $\alpha_{s}=1.5$, $\alpha_{r}=1.3 s_{r}=2 \cdot 10^{-4} s_{s}=5 \cdot 10^{-4}$ and additionally the values of the mass $m_{1}=0.07 m_{2}=0.05 \quad m_{3}=0.03$ $m_{4}=0.05 \quad(\mathrm{~kg})$ and the angles $\beta_{1}=0.29 \mathrm{rad}$, $\beta_{2}=0.443, \beta_{3}=0.608, \beta_{4}=0.796 \quad(\mathrm{rad})$ and the angular acceleration for descending motion were found.

$$
\begin{aligned}
& \varepsilon_{1}=35.123 ; \\
& \varepsilon_{2}=22.765 ; \\
& \varepsilon_{3}=7.51 ; \\
& \varepsilon_{4}=15.254 .
\end{aligned}
$$

Now, the values of the accelerations $\varepsilon_{k}$ and masses $m_{k}$ are introduced in the equations 20 with the unknowns $\alpha_{s}$ and $\alpha_{r}$ and the solution obviously must be:

$$
\alpha_{s}=1.5, \alpha_{r}=1.3
$$

$$
\left[\begin{array}{l}
\varepsilon_{1} \\
\varepsilon_{2} \\
\varepsilon_{3} \\
\varepsilon_{4}
\end{array}\right]=\left[\begin{array}{l}
35.088 \\
22.765 \\
7.51 \\
15.254
\end{array}\right]
$$

$$
\begin{aligned}
& \mathrm{x}:=1.5 \quad \mathrm{y}:=1.5 \\
& \text { Given } \\
& \mathrm{H} 1(\mathrm{x}, \mathrm{y})=0 \quad \mathrm{H} 2(\mathrm{x}, \mathrm{y})=0 \\
& \text { find }(\mathrm{x}, \mathrm{y})=\left(\begin{array}{l}
1.497 \\
1.34
\end{array}\right)
\end{aligned}
$$

Considering that the angular acceleration $\varepsilon_{1}$ was found with a $0.2 \%$ error, it is obtained (from the Mathcad program written for the study) that:

$$
\left[\begin{array}{l}
\varepsilon_{1} \\
\varepsilon_{2} \\
\varepsilon_{3} \\
\varepsilon_{4}
\end{array}\right]=\left[\begin{array}{l}
35.159 \\
22.765 \\
7.51 \\
15.254
\end{array}\right]
$$

$$
\begin{aligned}
& \mathrm{x}:=1.5 \quad \mathrm{y}:=1.5 \\
& \quad \text { Given } \\
& \mathrm{H} 1(\mathrm{x}, \mathrm{y})=0 \quad \mathrm{H} 2(\mathrm{x}, \mathrm{y})=0 \\
& \text { find }(\mathrm{x}, \mathrm{y})= \\
& \text { did not find solution }
\end{aligned}
$$

To clarify this behaviour, the variations of the two function from system 21 were represented for values of $\alpha_{s}$ and $\alpha_{r}$ in the range $(1,2)$. The two functions are represented in Fig. 4 by the level curves. 


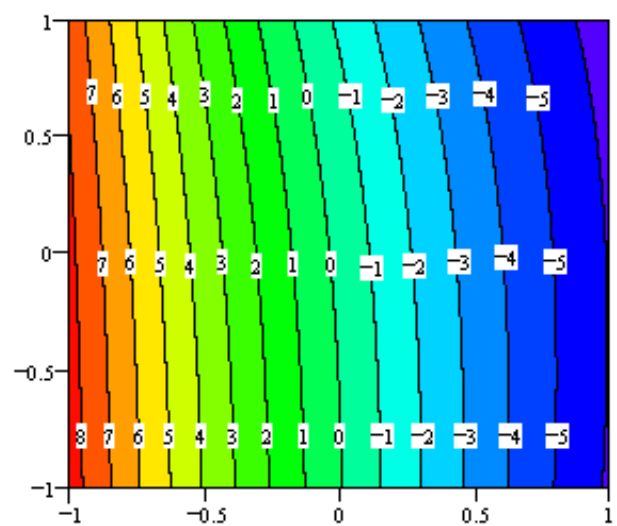

$\mathrm{H} 1$

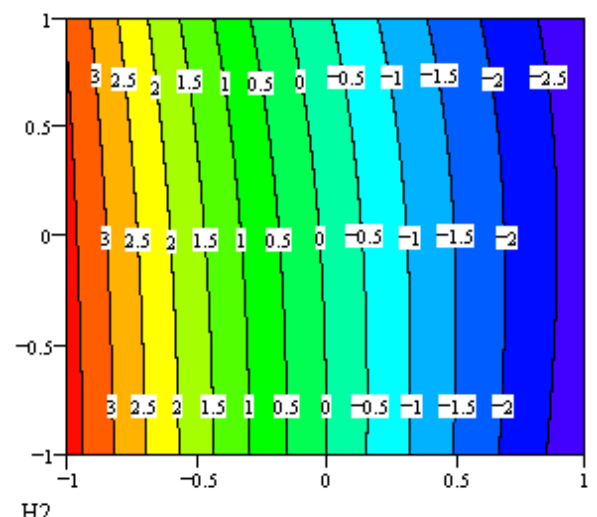

$\mathrm{H} 2$

Fig. 4. The functions $H_{1} ; H_{2}$ plotted by level curves

As it can be noticed form Fig. 4, the curves $H_{1}=0$ and $H_{2}=0$ are very closed to each other and it is difficult to identify the point of intersection.

The following function is defined:

$$
H=\sqrt{H_{1}^{2}+H_{2}^{2}}
$$

If the system 21 has a stable solution the plot of the function $H$ should present distinct minimum points.

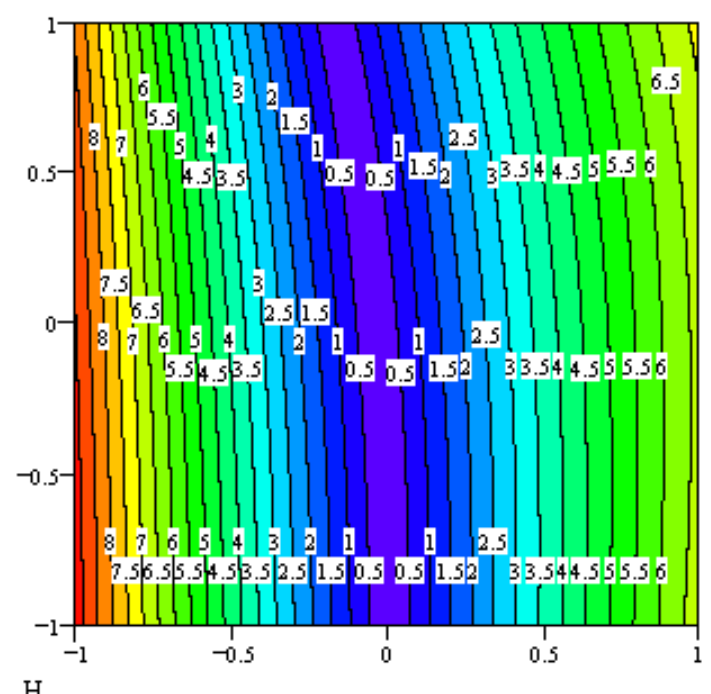

Fig. 5. Representation of the $H$ function
As it can be observed from Fig. 5, the region where the $H$ presents values close to zero is very wide on one direction and this fact justifies the instability of the solution.

Next, the above methodology applied to a problem with a stable solution is presented. More exactly, it is considered a problem where the functions $H_{1}$ and $H_{2}$ have simple expression, so the solution of the system 21 can be obtain analytically.

The problem of finding the intersection between a circle and an ellipse is considered. The equation of the circle is:

$$
H_{1}(x, y)=x^{2}+y^{2}-r^{2}=0
$$

where $r$ is the radius of the circle. The equation of the ellipse is:

$$
H_{2}(x, y)=\frac{x^{2}}{a^{2}}+\frac{y^{2}}{b^{2}}-1=0,
$$

where $a, b$ are the ellipse semi-axes. The graphical solution of the problem is presented in Fig. 6.

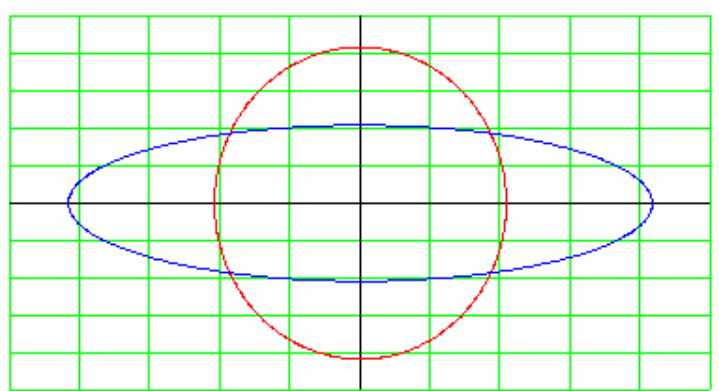

Fig. 6. The graphical solution of the problem

Next, in Figs. 7 and 8 the contour plots of the functions $H 1$ and $H 2$ (relations 25 and 26, respectively) are traced.

The contour lines of function $\mathrm{H}$ are plotted in Fig. 9. Due to the existence of stable solutions of the problem, the domains from $x y$ plane where the solutions should be looked for are well defined

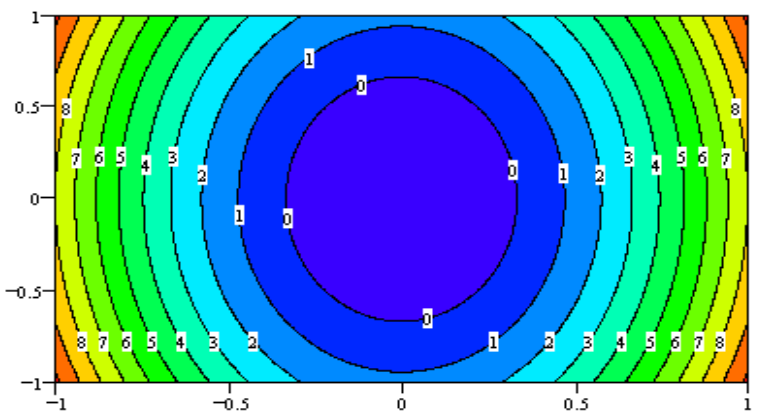

Fig.7. Contour plot of $\mathrm{H} 1$ function 


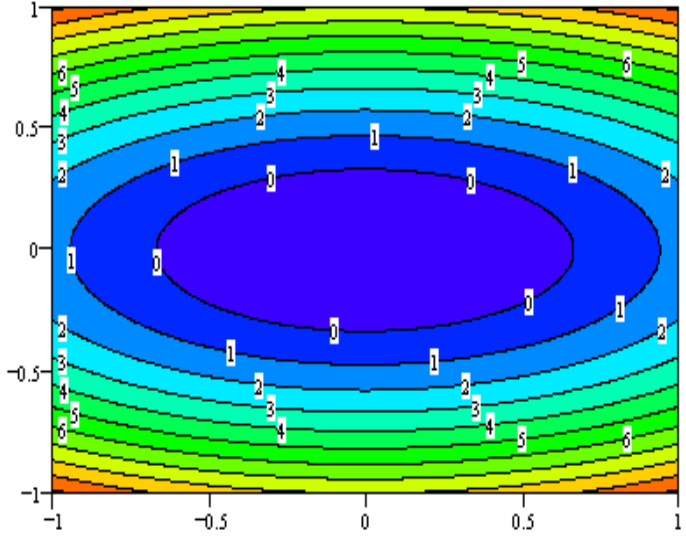

$\mathrm{H} 2$

Fig.8. Contour plot of $\mathrm{H} 2$ function

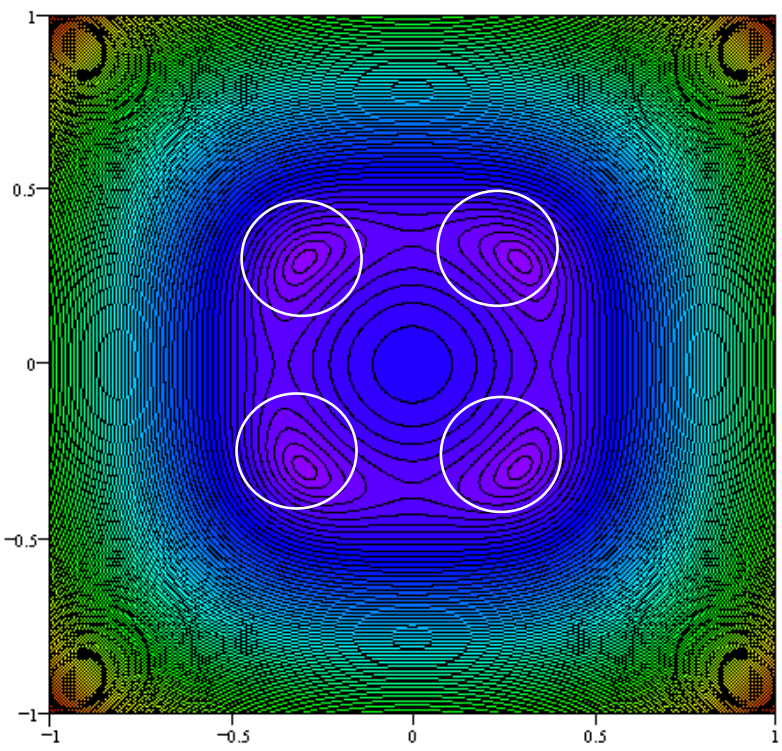

$\mathrm{H}$

Fig. 9. The contour plots of $\mathrm{H}$ function and the domains of the solutions

\section{Conclusions}

The paper shows that when a bearing ball is contacting two identical metallic parallel rods, two contact points occur and here there are present both spinning and rolling friction torques. It is assumed that each of the two moments depends on the normal force according to a power law relation and four parameters are necessary for characterizing the two moments: the coefficients and the exponents from the two relations moment-normal force.
The method proposed in order to establish these parameters is finding the acceleration of the ball for downward motion for four different inclination angles of the plane of the two rods. Consequently, a system of four equations with four unknowns is obtained. The system is linear with respect to the coefficients of spinning and rolling friction allows for reducing the system to a linear one with two unknowns, the two exponents.

For an actual case, when the characteristics of the system and the values of the four unknowns were assumed identified, it is proved that the system is extremely instable. Practically, an insignificant variation of the known parameters produces a substantial variation of the solution.

This work was supported by a grant of the Romanian Ministry of Research and Innovation, CCCDI - UEFISCDI, project number PN-III-P1-1.2-PCCDI-2017-0404 / 31PCCDI / 2018 , within PNCDI III".

\section{References}

1. K. L. Johnson, Contact Mechanics (Cambridge University Press 1985)

2. F. Schmelz, H.-C. Seherr-Thoss, E. Aucktor, Universal Joints and Driveshafts, Analysis, Design, Applications (Springer 2006)

3. N. Alves, M. T. Peixinho, P. Silva, P. Flores, H. M. Lankarani, Mech. and Mach. Theory, 85, 172 (2015)

4. V. L. Popov, Contact Mechanics and Friction. Physical Principles and Applications (Springer 2017)

5. G. P. Cherepanov, J. Appl. Mech. Techn. Physics 55 (1) 182 (2014)

6. S. Alaci, F. C. Ciornei, D. Amarandei, L. Irimescu, I. C. Romanu, M. A. Rotar, IOP Conf. Ser. - Mater. Sci. Eng. 174012008 (2017)

7. M. C. Ciornei, S. Alaci, F. C. Ciornei., I. C. Romanu, IOP Conf. Ser. - Mater. Sci. Eng, 227, 012027 (2017)

8. S. Alaci, L. Irimescu, M. A. Popescu, D. Fodorcan, D. Amarandei, F. C. Ciornei, MATEC Web of Conf., 112, 07002 IManE\&E (2017)

9. F. C. Ciornei, S. Alaci, R. I. Bedrule, C. M. Drelciuc, Robotica \& Management 18, 2, 13 (2013)

10. S. Alaci, F. C. Ciornei, A. Ciogole, M. C. Ciornei, IOP Conf. Series: Materials Science and Engineering, 200, 012005 (2017)

11. S. Alaci, E. Diaconescu, F. C. Ciornei, L. Irimescu, D. Cerlinca, Proceedings Rotrib 10, 1 (2010) 